\title{
Féeries
}

Études sur le conte merveilleux, XVII $-\mathrm{XIX}{ }^{\mathrm{e}}$ siècle

\section{Barbe Bleue entre Charles Perrault et Amélie Nothomb. La réécriture littéraire comme miroir du monde sensible}

Blue Beard, between Charles Perrault and Amélie Nothomb. Literary Rewriting as a Mirror to the Sensitive World

\section{Isabella Mattazzi}

\section{OpenEdition \\ Journals}

Édition électronique

URL : http://journals.openedition.org/feeries/1449

ISSN : 1957-7753

Éditeur

UGA Éditions/Université Grenoble Alpes

Édition imprimée

ISBN : 978-2-37747-075-4

ISSN : 1766-2842

Référence électronique

Isabella Mattazzi, « Barbe Bleue entre Charles Perrault et Amélie Nothomb. La réécriture littéraire comme miroir du monde sensible », Féeries [En ligne], 15 | 2018, mis en ligne le 14 février 2019, consulté le 08 septembre 2020. URL : http://journals.openedition.org/feeries/1449

Ce document a été généré automatiquement le 8 septembre 2020

(c) Féeries 


\title{
Barbe Bleue entre Charles Perrault et Amélie Nothomb. La réécriture littéraire comme miroir du monde sensible
}

\author{
Blue Beard, between Charles Perrault and Amélie Nothomb. Literary Rewriting \\ as a Mirror to the Sensitive World
}

Isabella Mattazzi

\section{Bleu apprivoisé}

La Barbe bleue de Charles Perrault repose de toute évidence sur un dispositif visuel. La vision - douteuse, interdite, et enfin libérée et clairvoyante - est une constante sensorielle qui semble régler tous les équilibres parmi les personnages et qui, de fait, est le véritable moteur de la logique narrative du conte. Sans vision on n'aurait pas d'histoire. Sans vision on n'aurait pas de barbes, de femmes égorgées clouées aux murs, de clefs ensorcelées ou de frères bienveillants et bien équipés prêts à sauver l'héroïne de son mari meurtrier.

Même l'expérience du merveilleux, reléguée dans le conte aux seuls épisodes de la couleur de la barbe et de la clé sanglante qu'il est impossible de nettoyer ${ }^{1}$, semble relever d'un univers moins ontologique que sensoriel. Le sang sur la clé représente un péril seulement du moment qu'il va être $v u$ par Barbe bleue et donc, dans un jeu de triangulation de pulsions scopiques, du moment que, soumis à cette vision, il va dénoncer une autre vision préalable, à savoir le regard de la jeune épouse sur la chambre interdite et par conséquent sa culpabilité. Tous les éléments thématiques du conte, pour passer du silence immobile de leur présence textuelle à une condition de signification, doivent surgir d'un choc visuel en tombant sous le faisceau de lumière du regard du sujet. La clé sanglante ne serait porteuse d'aucun malheur si elle demeurait dans l'obscurité d'un tiroir, la chambre interdite pourrait garder son secret pour 
l'éternité si la jeune femme n'avait jamais regardé au-delà de la porte, les frères ne pourraient assumer leur fonction salvatrice nulle part sinon dans les yeux de leur sœur Anne dans l'un des crescendo visuels les plus réussis de l'histoire des contes littéraires.

\begin{abstract}
« Descends vite, ou je monterai là-haut. - Encore un moment, s'il vous plaît », lui répondait sa femme ; et aussitôt elle criait tout bas : Anne, ma sœur Anne, ne vois-tu rien venir?" Et la sœur Anne répondait: "Je ne vois rien que le soleil qui poudroie, et l'herbe qui verdoie." "Descends donc vite, criait la Barbe bleue, ou je monterai làhaut. - Je m'en vais ", répondait sa femme, et puis elle criait : « Anne, ma sœur Anne, ne vois-tu rien venir?" - Je vois, répondit la sœur Anne, une grosse poussière qui vient de ce côté-ci. - Sont-ce mes frères? - Hélas! non, ma sœur, c'est un Troupeau de Moutons. - Ne veux-tu pas descendre ? criait la Barbe bleue. - Encore un moment ", répondait sa femme ; et puis elle criait : "Anne, ma sœur Anne, ne voistu rien venir?" - Je vois, répondit-elle, deux cavaliers qui viennent de ce côté-ci, mais ils sont bien loin encore... Dieu soit loué, s'écria-t-elle un moment après, ce sont mes frères, je leur fais signe tant que je puis de se hâter².
\end{abstract}

2 Même la couleur particulière de la barbe du protagoniste masculin, élément d'inquiétante étrangeté doté d'une puissance assez forte pour phagocyter son identité (son nom) et le conte tout court (son titre), semble participer à ce jeu de cadrages perspectifs. Au tout début du texte, Perrault présente « un homme qui avait de belles maisons à la ville et à la campagne, de la vaisselle d'or et d'argent, des meubles en broderie, et des carrosses tout dorés", mais qui par malheur «avait la barbe bleue: cela le rendait si laid et si terrible, qu'il n'était ni femme ni fille qui ne s'enfuît de devant lui $»^{3}$. La richesse donc contre l'inquiétude visuelle. La raison économique contre la raison de l'œil ${ }^{4}$. Apparemment cette dernière semble primer : les femmes, au seul regard posé sur la barbe, s'échappent en faisant triompher la vérité de la sensibilité sur la vérité de la réflexion. Mais dans le cas d'une seule et unique fille, jeune cadette d'une "dame de qualité ", le regard projeté sur la barbe arrive lentement à modifier son côté troublant en réduisant le choc sensible de l'altérité de sa couleur, en amont des valeurs symboliques que pourrait recevoir cette condition d'anormalité physique. Si d'abord la jeune fille ne peut « se résoudre à prendre un homme qui eût la barbe bleue " (d'autant plus "qu'il avait déjà épousé plusieurs femmes, et qu'on ne savait ce que ces femmes étaient devenues »), après huit jours passés avec lui dans sa maison de campagne elle commence « à trouver que le maitre du logis n'avait plus la barbe si bleue, et que c'était un fort honnête homme $»^{5}$. L'apprivoisement de la Barbe bleue à l'égard de la jeune fille se joue donc sur une distorsion optique. Dans l'enchaînement syntagmatique, la perception précède toute réflexion : on perçoit cet homme comme monstrueux (et au second degré on le suppose avoir tué ses épouses), mais si sa barbe change de couleur, notre perception affective de cet homme s'en trouve aussi nécessairement changée, modifiant la signification morale de ses actions.

\title{
Bleu mélancolique
}

Bien des interprétations psychanalytiques du conte ont lu le texte comme l'histoire d'une progressive capacité de vision. Selon la leçon jungienne présentée par Clarissa Pinkola Estés, qui donne une lecture analytique et archétypale d'un corpus folklorique interprété comme un grand récit collectif de la féminité, la Barbe bleue, la sœur Anne et les frères ne seraient que des projections narratives des pulsions qui animent la structure psychique du sujet ${ }^{6}$. Selon cette perspective, la Barbe bleue, "prédateur naturel de la psyché », serait une instance autodestructrice du moi, force innée, 
incontournable, que seul un parcours articulé et complexe d'introspection analytique et de mise à jour de l'inconscient pourrait réduire au silence.

3 Cette lecture - tout comme celle de Bruno Bettelheim qui interprète le conte de Perrault à la lumière du sentiment de culpabilité lié à une « interdiction sexuelle ${ }^{7}$ »bien que convaincante ne semble pas prendre en considération l'aspect éminemment sensoriel mis en jeu par Perrault. S'il est vrai que la jeune protagoniste passe d'une condition de complète passivité victimaire à une pleine conscience de soi et donc achève son parcours initiatique, il est également intéressant de suivre les différentes étapes de ce parcours dans leur articulation sensorielle.

4 Il faut considérer d'abord la couleur de la barbe. Ute Heidmann, en inventoriant les références intertextuelles du conte de Perrault, renvoie pour le personnage de la Barbe bleue à la figure de « Portunus, dieu marin à la barbe bleue, serviteur fidèle de Venus et par ailleurs dieu des portes et des clés ${ }^{8} "$ que Perrault aurait repris d'Apulée mais, même au-delà des aspects certainement non secondaires de reconfiguration du texte par rapport à ses sources anciennes, il est indéniable que pour les lecteurs de l'époque la couleur bleue est un élément sémantiquement marqué. Le bleu porte sur soi, depuis toujours, la stigmatisation d'un élément hors norme, peu apprivoisé, peu commun. Aujourd'hui les couleurs qui nous entourent sont presque toutes synthétiques, produites industriellement, sans aucune différence symbolique qui puisse différencier une nuance de l'autre. Un tube de peinture jaune de cadmium et un tube de bleu outremer, dans un magasin spécialisé, ont le même prix et la même accessibilité au grand public. Mais il s'agit d'une conquête contemporaine, autrefois les couleurs n'avaient pas toutes la même disponibilité sur le marché et surtout le même prix. Le bleu d'outremer est la couleur la plus difficile à produire non synthétiquement. Il s'agit de la réduction en poudre d'une pierre semi-précieuse, le lapis-lazuli, qui depuis l'Antiquité arrive en Europe sur des navires marchands qui viennent d'un pays lointain, outre la Méditerranée. Pendant la Renaissance on appelle le bleu «Ultramarin » pour indiquer sa provenance, c'est-à-dire un pays imprécisé au-delà de la mer, l'actuel Afghanistan, d'où il arrive en suivant la route de la soie pour s'amarrer enfin à Venise, la place la plus importante en Europe pour ce qui concerne les biens exotiques. De plus, dans la nature, le lapis-lazuli se trouve mêlé avec d'autres minéraux et, avant son utilisation, il faut séparer le pigment des impuretés, il faut ensuite ajouter de l'huile, de la cire, de la résine, avec plusieurs passages complexes et très longs avant d'obtenir, par décantation, la poudre bleue. Le bleu se trouve donc, pour de simples questions techniques et géographiques, être depuis toujours la couleur la plus chère dans l'univers marchand occidental ${ }^{9}$. Ce n'est pas du tout une nuance neutre. C'est la couleur du lointain, de la richesse, de la complexité des savoirs, de ce qui n'est pas commun, de tout ce qui est - en un mot - Autre.

5 L'altérité est aussi question de complexion, de tempérament. Celui qui n'est pas à même de participer à la vie sociale porte en lui cette stigmatisation : ce sont les solitaires, les brutes, les hommes sauvages, les mélancoliques. Comme l'écrit Federica La Manna dans son essai sur la mélancolie au XVIII ${ }^{\mathrm{e}}$ siècle, "[l]'homme en tant que créature à moitié ange et bête, se trouve dans une condition de perpétuel équilibrisme. De l'ange il peut devenir brute, et plus il essaie de se rapprocher des dieux et d'une idée de perfection, plus fort est le péril qu'il en attire la vengeance, en tombant dans l'abysse de sa propre condition sauvage ${ }^{10} »$. L'homme mélancolique est alors l'homme-loup, l'homme ange et bête à la fois, celui qui est toujours à l'écart, qui préfère les bois et les forêts aux 
villages ${ }^{11}$. Dans la Grèce d'Aristote, l'homme qui souffre d'un flux trop abondant de bile noire (mélaina kolé) est aussi perittòs, fou, excessif, soumis à des déséquilibres perpétuels du corps et de l'humeur. Le mélancolique est celui dont les actions ne correspondent à aucune règle établie, mais il est surtout celui qui fait. Le nœud archaïque de la mélancolie est étroitement lié à une praxis bestiale : Ajax qui massacre les animaux en proie à un accès atrabilaire, et qui se suicide tout de suite après en se jetant sur son épée, est l'homme tout-praxis, il représente la totale perte de soi dans la violence du geste $^{12}$. Bien sûr, d'un point de vue iconographique, Ajax à un certain point de la narration s'assoit immobile, avec sa tête inclinée sur sa main, mais il s'assoit pour contempler les actions qu'il vient d'accomplir, sa folie demeure dans le geste, la contemplation de l'acte n'est qu'un passage successif. « Le héros des batailles d'Homère, écrit Marco Mazzeo, le protagoniste de la tragédie sophocléenne, un suicidé fortement connoté par les marques vivaces de la manie et du déséquilibre, devient ainsi le paradigme d'une typologie caractérielle différente, opposée : assis, pensif, prisonnier de ses doutes, il constitue désormais la représentation de l'action paralysée et de la régression à l'infini des pensées et des actions humaines ${ }^{13}$.»

La maladie du mélancolique, maladie de l'esprit et affliction du corps, est une maladie bleue. Si le nom Mélancolie-bile noire (Mélaina kolé) nous conduit dans l'univers visuel du noir avec sa connotation dysphorique, selon Agrippa, dans son De occulta philosophia, " un mélange entre flegme et mélancolie, avec une prépondérance de mélancolie produirait une couleur bleuâtre ${ }^{14}$ ", tout comme pour Goethe, dans sa Théorie des couleurs, « le bleu azur, fils du noir, est la couleur du froid, de la tristesse, de l'ombre ${ }^{15}$ ». La couleur de la barbe du personnage de Perrault donc n'est pas une couleur quelconque, mais renvoie à des significations symboliques qui traversent la culture occidentale depuis des siècles ${ }^{16}$. Dans les différentes versions orales du conte, d'autre part, comme le souligne Catherine Velay-Vallantin, «on ne l'a pas toujours nommé Barbe-Bleue, mais également Barbe-Verte (Vendée, Anjou) ou Barbe-Noire (La Chapelle d'Apchier) ou encore Barbe de bleu (Louisiane). Derrière l'usage ludique des surnoms interchangeables, s'alignent ici les trois couleurs, bleu, vert et noir, qui délimitent, dès le Moyen Âge et selon un héritage ancien (Rome et Byzance), le concept de sombre, du deuil, de la folie, du désordre ${ }^{17}$ ». Le bleu de la barbe est un symptôme, c'est un message parfaitement compréhensible pour les contemporains de Perrault. Face à cette évidence visuelle, la jeune cadette opère alors un obscurcissement sensoriel, au fur et à mesure qu'elle passe ses huit journées avec Barbe bleue elle efface la "bleuité » du message, en altérant sa perception et par conséquent sa connaissance du monde (il ne faut pas oublier que tout le siècle suivant confiera à une métaphore visuelle son plus haut espoir d'une nouvelle fondation de la connaissance), jusqu'à arriver à une sorte de degré zéro de la vue : le noir qu'elle va rencontrer à l'ouverture de la chambre interdite en bas de l'escalier.

D'abord elle ne vit rien, parce que les fenêtres étaient fermées; après quelques moments elle commença à voir que le plancher était tout couvert de sang caillé, et que dans ce sang se miraient les corps de plusieurs femmes mortes et attachées le long des murs ${ }^{18}$.

\section{Bleu/rouge}

La jeune fille entre dans la chambre, l'espace est noir, la vue complètement empêchée par les fenêtres fermées. Son parcours de distorsion optique - commencé par une 
édulcoration de la couleur bleue et une méconnaissance du message contenu dans sa symbolique visuelle, suivies d'un progressif décalage entre sensation et conceptarrive donc à son comble.

7 Or, neuf ans avant la publication des Contes de ma mère l'Oye, William Molyneux et John Locke s'échangent par lettre les destins de l'un des noyaux fondamentaux de la pensée théorique du siècle à venir. Le problème de Molyneux les intéressera plus ou moins tous : de Berkeley à Leibniz, de La Mettrie à Voltaire, en passant par Condillac, Diderot, Bonnet, jusqu'à Kant. Claude Perrault, le frère du conteur, n'est pas en reste dans cette effervescence ${ }^{19}$. Dans l'image d'un homme né aveugle qui se trouve face à un cube et à une sphère, se croisent la réflexion sur les possibilités et les limites de la connaissance humaine, les études sur les origines des idées, le débat sur le rapport entre sensation, images et concept, mais surtout la conception de la vue comme le véhicule privilégié d'une démonstration exemplaire des articulations multiples du rapport entre raison et sensibilité. Au sein de la culture des Lumières, le paradigme visuel semble bénéficier d'une hégémonie incontestée. Voir c'est connaître, écrit Diderot dans ses Éléments de physiologie ${ }^{20}$ et pour Potocki les yeux sont des lentilles intellectives au moyen desquelles on peut penser la réalitée ${ }^{21}$. Si on suit cette perspective, la jeune mariée semble vivre avec son mari dans un état de complet dérèglement sensible et intellectuel à la fois, elle ne comprend rien de la réalité qui l'entoure parce qu'elle ne voit littéralement rien.

8 Mais dans le noir de la chambre interdite, enfin, lentement les images commencent à prendre un contour défini. Encore une fois, plus que des formes et des volumes, ce sont des couleurs qui viennent frapper les yeux de la jeune femme. C'est le sang qui apparaît en premier, élément fluide, instable, dont l'identité se révèle dans l'immédiateté visuelle de sa couleur rouge. Le rouge du sang, qui se situe exactement à l'opposé du bleu dans l'échelle de la température des couleurs (froids-chauds), nous porte dans un univers diamétralement opposé à la symbologie dysphorique du début du conte : c'est le retour à la vie, bien qu'à travers une image de mort.

Cependant, le retour à la pleine maîtrise de la sensibilité doit être accompli graduellement : la vision libérée, l'exacte mise au point de l'œil et de la connaissance n'est pas immédiate. Le rouge du sang sur le plancher arrive le premier, et tout de suite après Perrault nous informe que "dans ce sang se miraient les corps de plusieurs femmes mortes ». La jeune épouse semble donc voir le reflet des femmes qui l'ont précédée juste un instant avant de voir leurs corps, et elle parvient ainsi à prendre conscience de l'action destructrice du loup mélancolique (et de la réalité tout court) après un parcours en quatre étapes : perception de la couleur, perception de l'image (bidimensionnelle), perception de l'objet (volumétrique), réflexion (condamnation morale du tueur en série). Encore une fois, tout comme au début du conte, la pensée est complètement asservie à la sensation (qu'elle soit juste ou fausse). Si auparavant le bleu apprivoisé de la barbe pouvait annoncer l'honnêteté de l'homme qui la portait, désormais le rouge des femmes clouées au mur transforme cet honnête homme en assassin. C'est ce passage visuel d'un extrême à l'autre de la gamme froid/chaud (mort/ vie), et seulement lui, qui permet d'atteindre enfin la conclusion de l'histoire, avec un véritable triomphe du regard, cette fois-ci confié à Anne la sœur qui, avec ses yeux grand ouverts vers l'horizon du haut de la tour, peut témoigner de la conclusion heureuse du parcours initiatique de la cadette. La capacité sensorielle du sujet est enfin rétablie et l'on a retrouvé un parfait alignement entre connaissance sensible et discernement. La Barbe bleue est reconnu dans toute la portée violente de sa «bleuité » 
et il ne peut payer qu'avec sa mort, tout comme Ajax, sa position marginale dans l'organisme social.

\section{Bleu mythopoïétique}

Le dispositif visuel de Perrault a traversé les siècles dans un processus d'adaptation et de diffusion qui a intéressé presque tous les domaines artistiques : l'opéra, la télévision, le cinéma, le théâtre, la musique et, naturellement, la littérature ${ }^{22}$. La Barbe bleue semble posséder toutes les caractéristiques exigées par la mythocritique et la mythanalyse des dernières années pour paraître à plein titre parmi les figures du mythe littéraire moderne. Le pattern "barbe bleue», en plus d'être transhistorique, porteur d'une vérité à la fois universelle, naturelle et transcendante ${ }^{23}$, possède la forme d'un «récit fermement structuré, symboliquement surdéterminé, d'inspiration métaphysique, reprenant le syntagme de base d'un ou de plusieurs textes fondateurs ${ }^{24}$ " qu'André Siganos déclare incontournable pour la fondation de tout mythe littéraire. Son histoire se situe comme élément générateur de sens à l'intérieur d'une série presque infinie de réécritures selon ces principes de "flexibilité », d' " irradiation » et de "focalisation multiple ${ }^{25}$ appelés par Pierre Brunel à sous-tendre une nouvelle mythologisation du discours contemporain. La Barbe bleue, homme-bête, nouveau Minotaure, est au cœur d'une série de métamorphoses sans solution de continuité, est le centre d'une tension dialectique ouverte vers l'infini.

10 Toute réécriture est une transfiguration qui contraint à un double regard, un regard figé sur le hic et nunc du texte contemporain, et en même temps tourné vers l'arrière, vers la source primitive et les codes de la culture qui l'a générée. « La réécriture d'un texte, affirme Enrico Guglielminetti, opère une refonte du texte avec un transfert de forme à forme, du texte source à un texte nouveau. Dans la réécriture le texte est un hypertexte, ouvert à un nombre variable de textes virtuels. L'hypertexte est en ce cas extensionnel, c'est-à-dire que la réécriture du texte se produit dans un lieu différent de celui qu'il occupait à son origine. La réécriture est donc une métamorphose vitale du texte $^{26}$. " Dans cette métamorphose vitale, les aspects structurels du texte-source sont soumis à une torsion continuelle qui annonce leur mort dans la transformation, mais en même temps révèle leur irréductibilité. Le dispositif de la vision de La Barbe bleue se trouve chaque fois contredit et renouvelé par chacune de ses métamorphoses vitales, modifié et réaffirmé, vaincu et triomphant.

\section{Bleu disparu}

Dans Barbe bleue d'Amélie Nothomb, roman paru en 2012, la réécriture semble présenter un décalage évident par rapport au texte-source. Seul le paratexte avoue le lien avec Perrault. Sans le titre nous resterions dans l'ignorance de nous trouver face à une reformulation du mythe du tueur en série.

Quand Saturnine arriva au lieu du rendez-vous, elle s'étonna qu'il y ait tant de monde. Certes, elle s'était doutée qu'elle ne serait pas l'unique candidate : de là à être reçue dans une salle d'attente où quinze personnes la précédaient, il y avait de la marge ${ }^{27}$.

11 Si dans La Barbe bleue de Perrault la protagoniste féminine répondait à des appellatifs génériques (la cadette, la fille...), ici elle bénéficie d'une véritable identité. Elle possède un prénom et un nom : Saturnine Puissant. La présence d'un nom propre apporte une 
première torsion du pattern. Appeler un personnage par son nom équivaut à lui donner un poids spécifique non négligeable dans les équilibres des forces symboliques du roman. De plus, pour Nothomb, l'assignation d'un prénom n'est jamais anodine, les noms de ses personnages sont des traces, les éléments d'un paradigme indiciaire qui permet au lecteur de construire une histoire supplétive au-delà de la structure même de la narration. En ce cas, la cadette passive et ingénue de Perrault est devenue une femme toute-puissante au prénom à la forte dominante masculine. Le prénom Saturnine tire son origine d'un dieu violent qui a émasculé son père et dévoré ses fils : Saturne, le seigneur du temps et de la mélancolie. Dans l'étude monumentale de Klibansky, Panofski et Saxl, Saturne et la mélancolie ${ }^{28}$, Saturne-Chronos est l'image à travers laquelle on peut lire la civilisation occidentale comme le produit d'un rayonnement linéaire d'un sujet artistique réflexif et créateur à la fois. Dans cette étude iconologique sur la gestualité mélancolique (depuis l'Athéna mélancolique ou l'Alcmène sur le bûcher de la Grèce classique, jusqu'à la modernité de Munch et de Sérusier, en passant bien évidemment par l'archétype de Dürer), Ervin Panofski présente une dualité du sujet mélancolique qui semble traverser les époques en se déclinant sur les axes cartésiens de la folie et de la connaissance. La tête inclinée sur son bras de l'ange de Dürer est le geste de l'homme fasciné par le gouffre de la tristesse et de l'abandon, mais c'est aussi le geste des devins, des prophètes, des penseurs, de ceux qui ont accès à la connaissance, en un mot de ceux qui «voient » la réalité d'un regard plus éclairé que les autres.

Le mot Saturnine renvoie aussi au saturnisme, intoxication aiguë ou chronique par le plomb provoquant une altération du système nerveux (troubles neuromoteurs, retard mental...) et ensuite la mort. La médecine fait référence là encore au dieu Saturne car, dans la pratique alchimique, la planète qui lui est dédiée est le symbole du plomb. Pour les alchimistes le plomb, métal lourd et imparfait, représente le premier degré - l'état de nigredo - d'un processus triadique d'élévation intellectuelle. " Transformer le plomb en or, selon Annick De Souzenelle, est l'exact travail de croissance de l'homme, celui que commence d'opérer le chevalier, ou tout être qui vit sa première naissance intérieure ${ }^{29}$. " La planète Saturne, dont le mouvement de circonvolution est le plus lent de tout le système solaire, conjugue aussi le plomb à la lourdeur et à la lenteur traditionnellement associées à l'humeur mélancolique. Si nous pensons à des locutions comme "une tristesse de plomb", ou "se sentir les jambes en plomb", on peut percevoir la persistance de cette conception dans le langage d'aujourd'hui.

Ainsi, dès la première ligne du texte, l'héroïne de Nothomb semble présenter, dans la poignée de voyelles et de consonnes qui disent son identité, un réseau complexe d'échos et de renvois. Si d'une part elle se trouve à l'opposé de l'hérö̈ne de Perrault par son évident état de "puissance", de l'autre elle semble partager avec celle qui l'a précédée un même parcours de croissance et d'affinement perceptif. Son état de nigredo - la quantité de plomb contenue dans son prénom - nous indique un sujet sur le point d'entamer un parcours à peine ébauché ; elle est dans une condition de connaissance et de « vision » imparfaites que la rencontre avec Barbe bleue pourra seule faire évoluer.

Dans le conte de Perrault, le lien entre les deux personnages principaux était en premier lieu de nature sexuelle, indissolublement associé à l'institutionnalisation de la sexualité (on a affaire à un contrat de mariage) et à ses tabous. Dans le roman de Nothomb, la sexualité fait place à l'économie, on a affaire à un contrat, mais cette foisci à un contrat de location. On est à Paris, de nos jours, et une jeune fille du nom de 
Saturnine Puissant répond à une annonce pour une chambre à louer dans le $\mathrm{VI}^{\mathrm{e}}$ arrondissement :

Deux heures plus tard, un secrétaire la conduisit dans un bureau gigantesque, orné d'admirables fleurs mortes. De l'homme qui lui serra la main, la jeune femme ne vit qu'une chose: il avait l'air d'un dépressif profond, le regard éteint et la voix épuisée.

- Bonjour mademoiselle, je suis don Elemirio Nibal y Milcar, j'ai quarante-quatre ans ${ }^{30}$.

15 Si la protagoniste féminine a acquis par son nom un barycentre identitaire bien solide, le protagoniste masculin à l'inverse semble avoir perdu le sien. Dans cette première description de don Elemirio Nibal y Milcar il n'y a plus de barbe ni aucune connotation de «bleuité », aucune trace non plus d'inquiétante étrangeté sur son visage. Ou mieux, la «bleuité » de la barbe est devenue maintenant une «beauté » mélancolique, car cet homme possède toutes les caractéristiques de la maladie atrabilaire. Il a l'air d'un "dépressif profond », c'est un homme hors norme, hors de la société, hors du temps. Il est un aristocrate, un grand d'Espagne, il appartient à un lieu et à un temps lointains. Il ne sort jamais de chez lui, il ne fréquente personne, il ne travaille pas car sa position économique lui permet de vivre de ses rentes. Il n'y a que peu de passions qui le tiennent en vie : la cuisine, la théologie et la photographie.

Enfin il la mena jusqu'à une porte peinte en noir.

- Ceci est l'entrée de la chambre noire où je développe mes photos. Elle n'est pas fermée à clef, question de confiance. Il va de soi que cette pièce est interdite. Si vous y pénétriez je le saurais et il vous en cuirait $^{31}$.

16 Si le motif de la barbe bleue a disparu, celui de la chambre interdite, en revanche, demeure central. Le noir des fenêtres fermées du texte de Perrault a trouvé une structuration en assumant la forme volumétrique d'une chambre de développement de l'image, c'est-à-dire d'un lieu technique de production et de gestion de la vision. Ici il n'y a pas de clé, l'accès au dispositif visuel est libre, mais ce libre accès aura, de toute façon, un prix. La porte de la chambre noire est dotée d'un dispositif cryogénique, à chaque fois que quelqu'un entre dans la pièce sans avoir préalablement déconnecté le minuteur du dispositif, la porte se referme sur elle-même sans pouvoir être ouverte de l'intérieur, la température de la pièce rejoint lentement des valeurs glaciales et la chambre se transforme en un lieu d'agonie et de mort.

17 À partir de ce point de départ - une jeune fille saturnienne, puissante et impuissante à la fois, un homme mélancolique soumis lui aussi à cette même condition, un dispositif visuel de développement de l'image (et de la connaissance) - , le texte se construit comme un véritable duel perceptif. Les deux contendants se retrouvent tous les soirs de part et d'autre de leur échiquier personnel - la table de l'appartement où ils partagent leur dîner - pour une partie qui se joue autour d'une énigme dont la résolution semble venir du cœur du monde sensible. Le thème dominant du Barbe bleue de Nothomb est encore une fois la couleur. Une couleur, cette fois-ci, non seulement déclinée le long d'une opposition binaire rouge/bleu, mais articulée à travers toutes les nuances du spectre de la lumière.

\section{Bleu-or}

- [...]. Il s'agit d'une crème à base de jaunes d'œufs.

- Vous me servez une omelette et puis des œufs en guise de dessert?

- J'ai une passion théologique pour les œufs. [...] 
- Donnez m'en donc la valeur d'un coquetier.

L'hôte alla chercher des tasses en or massif et les remplit d'une onctuosité jaune. Saturnine en resta figée d'éblouissement.

- Ce jaune opaque dans cet or baroque, c'est d'une beauté ! finit-elle par dire.

Don Elemirio, pour la première fois, regarda la jeune femme d'un intérêt véritable.

- Vous êtes sensible à cela?

- Comment ne pas l'être? Rouge et or, bleu et or, même vert et or sont des associations sublimes, mais classiques. Jaune et or, en art, cela n'apparaît pas. Pourquoi? C'est la couleur même de la lumière, modulée du plus mat au plus brillant.

L'homme posa sa cuillère et avec toute la solennité possible déclara :

- Mademoiselle, je vous aime ${ }^{32}$.

L'or est la première pièce que le nouveau Barbe bleue pose sur l'échiquier du texte. L'or en tant que couleur, mais aussi en tant que saveur (le champagne que l'on boit pendant tout le roman), et aussi en tant qu'expérience du toucher (l'étoffe de la jupe jaune-or qu'il coupe de ses propres mains et dont il fait cadeau à Saturnine) ${ }^{33}$. La couleur est donc la représentation métonymique de la sensation, la vision étant capable d'englober en ce cas tous les autres sens. Valeur sensible totale, la couleur est aussi une valeur morale et une monnaie d'échange sentimental : face à la déclaration d'admiration de la jeune fille pour la juxtaposition or-jaune, don Elemirio découvre et révèle son inclination pour elle (je vous aime). Exactement comme la cadette de Perrault avait accepté en mariage un homme à cause de la normalisation du ton de bleu de sa barbe, don Elemirio tombe amoureux de sa locataire seulement après un court-circuit sensoriel. Saturnine est associée à une couleur qui devient subitement une marque identitaire. Elle deviendra dorénavant la fille d'or, aux yeux de son loueur amoureux elle sera la représentation vivante d'une sensation visuelle ${ }^{34}$.

La couleur est aussi la clé de la résolution de l'énigme du roman. Avant l'arrivée de Saturnine, huit femmes ont répondu à l'annonce concernant la chambre de l'appartement de don Elemirio et ont signé le même bail de location. Avec Saturnine, les femmes de la Barbe bleue sont devenues neuf, exactement comme les sept couleurs du spectre élargies au blanc et au noir constituant la présence ou l'absence absolue de lumière. À chacune de ses femmes, la Barbe bleue de Nothomb a associé une couleur précise, il a fabriqué pour elles des vêtements de cette même couleur, il a cuisiné des plats avec une précision de peintre, en collant leur identité sur une monodimensionnalité sensorielle sans voie de secours. Pour lui, la sensation visuelle, comme perception totalisante de la couleur, est le seul moyen de compréhension de la réalité, il n'y a qu'une vision toute puissante et totale (vision à laquelle participent le goût, l'odorat, le toucher et l'ouie) qui puisse atteindre l'objet de sensation dans son essence primitive. Les femmes-couleurs deviennent donc la déclinaison d'un univers conçu comme un catalogue du monde sensible, et la chambre noire, comme une Encyclopédie contemporaine, est la cage dans laquelle il devient possible de systématiser, d'ordonner et d'emprisonner à jamais la multiforme instabilité du réel. Si les philosophes du XVIII ${ }^{\mathrm{e}}$ siècle ont eu besoin de dix-sept volumes de texte et de onze volumes de planches pour une opération semblable, don Elemirio domine son propre univers grâce à une collection de photographies. Tour à tour les huit femmes, toutes vaincues par leur curiosité, sont entrées dans la chambre interdite, déclenchant le dispositif cryogénique et mourant ainsi de froid. À leur mort, don Elemirio a pris une photo en grandeur naturelle de leur cadavre vêtu de l'habit de leur couleur identitaire, et il a collé l'image aux murs de la pièce. 
- Quel est l'intérêt de photographier une morte?

- Le rôle de l'art est de compléter la nature et le rôle de la nature est d'imiter l'art.

La mort est la fonction que la nature a inventée dans le but d'imiter la photographie. Et les hommes ont inventé la photographie pour capter ce formidable arrêt sur l'image qu'est l'instant du trépas ${ }^{35}$.

Si, dans La Barbe bleue de Perrault la chambre interdite recelait à la fois des corps égorgés et leurs reflets sur la surface rouge du plancher, ici il n'y a que le reflet, dans une adhérence entre image et référent dont déjà Roland Barthes avait deviné l'essence spectrale : «Par nature, la Photographie (il faut par commodité accepter cet universel, qui ne renvoie pour le moment qu'à la répétition inlassable de la contingence) a quelque chose de tautologique : une pipe y est toujours une pipe, indéniablement. On dirait que la Photographie emporte toujours son référent avec elle, tous deux frappés de la même immobilité amoureuse ou funèbre, au sein même du monde en mouvement : ils sont collés l'un à l'autre, membre par membre, comme le condamné enchaîné à un cadavre dans certains supplices ${ }^{36}$. » Le rêve de la chambre noire du Barbe bleue de Nothomb est en effet celui d'une cristallisation de la réalité dans l'image. Dans cet univers d'exacerbation sensible portée à son comble, la sensation se trouve être complètement détachée de l'objet, l'image vit sans son référent en s'y substituant, et en l'éternisant dans une réduction de la complexité du vivant à une série de chocs perceptifs. Peu emporte au mélancolique don Elemirio de sortir de chez lui, de travailler, de vivre une vie active du moment qu'il se trouve face à l'éternisation de l'univers entier toutes les fois qu'il le désire.

21 Parmi ces huit photographies il y a malgré tout un espace vide. Une dernière place attend la dernière couleur afin que la palette soit complète. La dernière couleur du catalogue de don Elemirio, c'est celle que les alchimistes attribuaient à la condition de rubedo, dernier degré spirituel et cognitif auquel l'être humain puisse tendre: l'or. Lâme de plomb, enfin libérée de ses concrétions impures à travers un travail progressif et complexe, a finalement atteint sa perfection en se transmuant en le métal le plus précieux. La dernière photographie manquante signale la fin du parcours, c'est le sceau d'un équilibre immobile capable de faire de la chambre noire une parfaite reproduction, détachée et autonome, du monde extérieur. Mais ce parcours d'élévation et de perfectionnement a un prix. Saturnine, pour abandonner définitivement le plomb de son nom et devenir femme-or, pour accomplir la prophétie que son barbe bleudémiurge lui a imposée, ne peut que tuer, encore une fois, l'agent même de son perfectionnement, l'homme sans lequel elle n'aurait jamais pu terminer son parcours initiatique. La chambre cryogénique cette dernière fois se fermera sur son architecte qui, devenu Minotaure après avoir été Dédale, reste prisonnier de son propre labyrinthe d'images, car - exactement comme pour la jeune cadette de Perrault - le parfait alignement entre vision et connaissance, entre perception sensible et discernement doit forcément coïncider avec le meurtre de toute «bleuité " mélancolique, c'est-à-dire avec la renonciation du sujet au rêve que l'image seule pourrait enfin se substituer à la réalité.

Près de la station de métro, elle avisa un banc public et s'y assit pour l'attendre. Devant elle il y avait les Invalides dont la coupole venait d'être redorée à la feuille. Un éclairage idéal en rehaussait la lumière. La jeune femme eut tout le temps d'admirer cette splendeur.

À l'instant précis où don Elemirio mourut, Saturnine se changea en or ${ }^{37}$. 


\section{BIBLIOGRAPHIE}

\section{Bibliographie}

AGRIPPA Heinrich Cornelius, De Occulta Philosophia [1531], Potenza, Grenelle, 2015.

BARTHES Roland, La chambre claire, Paris, Gallimard/Seuil, 1980.

BETTELHEIM Bruno, The Uses of Enchantment: The Meaning and Importance of Fairy Tales, Londres, Thames \& Udson, 1976.

BRÉMOND Claude, «La barbe et le sang bleus ", dans Une nouvelle civilisation? Hommage à Georges

Friedmann, Paris, Gallimard, 1973, p. 355-366.

BRUNEL Pierre, Mythocritique. Théorie et parcours, Paris, PUF, 1992.

DiDEROT Denis, Éléments de physiologie [1774-1782], éd. P. Quintili, Paris, H. Champion, 2001.

GOETHE Johan Wolfgang von, La teoria dei colori. Lineamenti di una Teoria dei colori [1810], Milan, Il Saggiatore, 1981.

GuGLIELMINETTI Enrico, La metamorfosi nell'immobilità, Milan, Jaca Book, 2000.

FALCINELli Riccardo, Cromorama. Come il colore ha cambiato il nostro mondo, Turin, Einaudi, 2017.

GRMEK M. D. , « Un débat scientifique exemplaire : Mariotte, Pecquet et Perrault à la recherche du siège de la perception visuelle ", History and Philosophy of the Life Sciences, vol. 7, n² 2, 1985,

p. 217-255.

HEIDMANN Ute, « Expérimentation générique et dialogisme intertextuel : Perrault, La Fontaine, Apulée, Straparola, Basile ", Féeries, n 8, 2011, p. 45-69.

HeIDMANN Ute \& ADAm Jean-Marie, Textualité et intertextualité des contes. Perrault, Apulée, La Fontaine, Lhéritier, Paris, Classiques Garnier, 2010.

KLIBANSKY Raymond, PANOFSKI Erwin \& SAXL Fritz, Saturne et la mélancolie, Paris, Gallimard, 1989.

LA MANNA Federica, "Più solitario di un lupo". Tipologia del Melanconico nel Settecento tedesco, Lecce, Manni, 2002.

MAZZEo Marco, Melanconia e rivoluzione. Antropologia di una passione perduta, Rome, Editori Riuniti, 2012.

MEYNARD Jean-Albert, Le complexe de Barbe bleue. Psychologie de la méchanceté et de la haine, Paris, L'Archipel, 2006.

Nотномв Amélie, Barbe bleue, Paris, Albin Michel, 2012.

Perrault Charles, La Barbe bleue, dans Contes, éd. C. Magnien, Paris, Le Livre de Poche classique, 2006, p. 219-228.

PinKola Estés Clarissa, Women Who Run with the Wolves: Myths and Stories of the Wild Woman Archetype, New York, Ballantine Books, 1992.

Ротоскі Jean, Voyage dans l'Empire de Maroc fait en l'année 1791 [1792], dans J. Potocki, CEuvres, éd. Fr. Rosset et D. Triaire, Louvain, Peeters, 2004. 
SIGANOS André, Le Minotaure et son mythe, Paris, PUF, 1993.

SOUZENELLE Annick de, Le symbolisme du corps humain, Paris, Albin Michel, 1984.

STAROBINSKI Jean, Trois fureurs, Paris, Gallimard, 1974.

TATAR Maria, Secrets beyond the Door: The Story of Bluebird and his Wives, Princeton, Princeton University Press, 2004.

VELAY-VALLANTIN Catherine, «Barbe-Bleue : le dit, l'écrit, le représenté (XVIII $-\mathrm{XIX}^{\mathrm{e}}$ siècles) », Romantisme, $\mathrm{n}^{\circ}$ 78, 1992, p. 75-90.

ZYPES Jack, Why Fairy Tales Stick: The Evolution and Relevance of a Genre, New York, Routledge, 2006.

ZYPES Jack, « Un remake de La Barbe bleue, ou l'au-revoir à Perrault », Féeries, n 8, 2011, p. 71-90.

\section{NOTES}

1. Catherine Velay-Vallantin, dans son article «Barbe-Bleue : le dit, l'écrit, le représenté (xviII ${ }^{\mathrm{e}}$ XIX ${ }^{e}$ siècles) » (1992, p. 75-90), souligne l'existence d'autres éléments thématiques appartenant au domaine du merveilleux (comme les animaux sauvages apprivoisés par les capacités magiques de l'héroïne) présents dans les différentes versions orales du conte - notamment dans les versions bretonnes -, mais on ne fera référence ici qu'au texte établi par Perrault.

2. Ch. Perrault, La Barbe bleue, dans Contes, 2006, p. 226.

3. Ibid.

4. Claude Brémond, dans son article «La barbe et le sang bleus » (1973, p. 355-366), souligne combien le processus de "seigneuralisation» de Barbe-Bleue est tardif. Dans la version de Perrault, la Barbe bleue est simplement un homme "très riche " sans indication précise sur la caractérisation sociale du personnage. Son château et son "sang bleu » appartiennent à des stratifications successives.

5. Ch. Perrault, éd. citée, p. 219.

6. C. Pinkola Estés, Women Who Run with the Wolves: Myths and Stories of the Wild Woman Archetype, 1992.

7. B. Bettelheim, The Uses of Enchantment: The Meaning and Importance of Fairy Tales, 1976.

8. U. Heidmann, « Expérimentation générique et dialogisme intertextuel », 2011, p. 66. Voir aussi

U. Heidmann et J.-M. Adam, Textualité et intertextualité des contes. Perrault, Apulée, La Fontaine, Lhéritier, 2010.

9. Sur l'histoire du bleu, voir R. Falcinelli, Cromorama. Come il colore ha cambiato il nostro mondo, 2017, chapitre « Azzurro costoso. Coloranti e pigmenti prima della modernità ».

10. F. La Manna, "Più solitario di un lupo". Tipologia del Melanconico nel Settecento tedesco, 2002, p. 10 (notre traduction).

11. Pour les rapports entre mélancolie et lycanthropie, voir F. La Manna, ibid.

12. Sur la folie d'Ajax, voir J. Starobinski, Trois fureurs, 1974, et aussi M. Mazzeo, Melanconia e rivoluzione. Antropologia di una passione perduta, 2012.

13. M. Mazzeo, ouvr. cité, p. 147 (notre traduction).

14. H. C. Agrippa, De Occulta Philosophia [1531], 2015, p. 183 (notre traduction).

15. J. W. von Goethe, La teoria dei colori. Lineamenti di una Teoria dei colori [1810], 1981, p. 193 (notre traduction).

16. Une réminiscence de cette qualité dysphorique du bleu survit dans la langue anglaise dans certaines occurrences : feeling blue - être triste, blue Monday - le jour le plus triste de l'année...

17. C. Velay-Vallantin, art. cité, p. 79.

18. C. Perrault, éd. citée, p. 223. 
19. Voir M. D. Grmek, «Un débat scientifique exemplaire : Mariotte, Pecquet et Perrault à la recherche du siège de la perception visuelle », History and Philosophy of the Life Sciences, vol. 7, $\mathrm{n}^{\circ} 2$, 1985 , p. 217-255.

20. D. Diderot, Éléments de physiologie [1774-1782], 2001, p. 85.

21. J. Potocki, Voyage dans l'Empire de Maroc fait en l'année 1791 [1792], dans CEuvres, 2004.

22. Voir M. Tatar, Secrets beyond the Door: The Story of Bluebird and his Wives, Princeton, 2004 ; J. Zypes, Why Fairy Tales Stick: The Evolution and Relevance of a Genre, 2006 ; J. Zypes, « Un remake de La Barbe bleue, ou l'au-revoir à Perrault ", 2011, p. 71-89.

23. À propos de la Barbe bleue comme complexe névrotique, voir J.-A. Meynard, Le Complexe de Barbe bleue. Psychologie de la méchanceté et de la haine, 2006.

24. A. Siganos, Le Minotaure et son mythe, 1993, p. 32.

25. P. Brunel, Mythocritique. Théorie et parcours, 1992.

26. E. Guglielminetti, La metamorfosi nell'immobilità, 2000, p. 180 (notre traduction).

27. A. Nothomb, Barbe bleue, Paris, Albin Michel, 2012, p. 7.

28. R. Klibansky, E. Panofski et F. Saxl, Saturne et la mélancolie, 1989.

29. A. de Souzenelle, Le symbolisme du corps humain, 1984, p. 122.

30. A. Nothomb, ouvr. cité, p. 11.

31. Ibid., p. 12.

32. Ibid., p. 23-24.

33. «Saturnine rejoignit ses appartements et enleva sa jupe. C'est alors qu'elle remarqua la doublure du vêtement : don Elemirio avait choisi une étoffe jaune d'une délicatesse sans exemple. Elle se rappela son propos sur le jaune et l'or, et bien malgré elle, quelque chose grippa sa mécanique. Elle s'assit sur le lit et caressa la doublure. Une transe d'une subtilité déchirante s'empara d'elle. Elle retourna la jupe de manière à faire rendre gorge à ce jaune. L'habit écorché montra ses tripes sublimes. La douceur de ce tissu exaspéra les mains, puis les joues, de l'éberluée. » Ibid., p. 71-72.

34. Il est intéressant de remarquer que ce rôle de pleine identification entre couleur et personnage, dans le texte de Perrault, est réservé à la Barbe bleue.

35. Ibid., p. 97.

36. R. Barthes, La chambre claire, 1980, p. 12.

37. A. Nothomb, ouvr. cité, p. 125.

\section{RÉSUMÉS}

Dans cet article est adoptée une approche intertextuelle pour comprendre comment le canon littéraire occidental a acquis une physionomie définie par la permanence et la métamorphose de textes fondateurs et par leur circulation, leur réception et leur appropriation au-delà de leur contexte. Les œuvres littéraires, qui sont lues, traduites et transposées d'une culture à l'autre, sont essentielles pour étudier les processus d'écriture créative et la multiplicité ou la diversité de leur auditoire. Dans le cas envisagé, la réécriture d'Amélie Nothomb du mythe littéraire de Barbe bleue, à partir du texte de Charles Perrault, semble offrir une clé non secondaire pour comprendre la culture qui a engendré ce mythe et la culture qui a reçu le texte métamorphosé, dans un rapport complexe avec l'univers de la perception et ses différentes articulations. 
In this article an intertextual approach to literature is adopted to understand how the western canon has come to acquire a physiognomy defined by the permanence and the metamorphosis of founding literary texts and by their circulation, reception and appropriation beyond their socialhistorical context. Literary works, which are read, translated and translocated from one culture to another are key to understanding the processes of creative writing and the multiplicity or diversity of their audience.

In this case, Amélie Nothomb's rewriting of the literary myth of Blue Beard from Charles Perrault's text seems to offer a non-secondary key to understanding the culture that engendered this myth and the culture that received the new text, through their complex relation to the universe of perception and its different articulations.

\section{INDEX}

Mots-clés : Charles Perrault, Amélie Nothomb, Barbe bleue, réécriture, métamorphose, mélancolie, théorie des couleurs, dispositifs visuels, alchimie, mythopoï̀se

Keywords : Charles Perrault, Amélie Nothomb, bluebeard, rewrite, metamorphosis, melancholy, color theory, visual devices, alchemy, mythopoiesis

\section{AUTEUR}

ISABELLA MATTAZZI

Université de Ferrara 